LC-MS analysis of oils of Monodora myristica and Monodora tenuifolia and isolation of a novel cyclopropane fatty acid

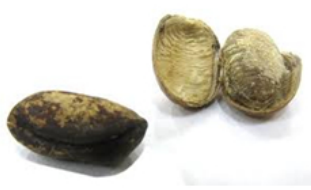

Monodora myristica

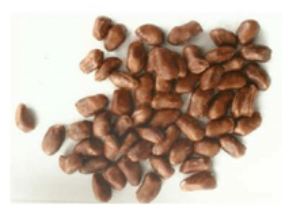

Monodora tenuifolia
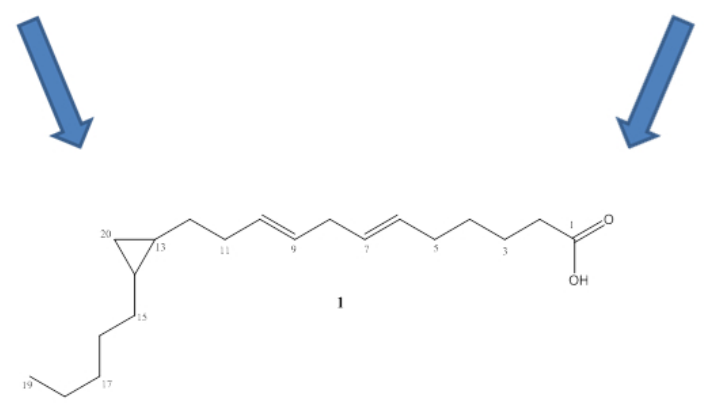

Structure of isolated cyclopropane fatty acid. 


\title{
LC-MS analysis of oils of Monodora myristica and Monodora tenuifolia and isolation of a novel cyclopropane fatty acid
}

\author{
Al-Tannak, N.F ${ }^{1,2^{*}}$, Ibrahim Khadra ${ }^{2}$, Igoli N.P and Igoli J.O ${ }^{4}$ \\ ${ }^{1}$ Department of Pharmaceutical Chemistry, Faculty of Pharmacy, Kuwait University. \\ ${ }^{2}$ Strathclyde Institute of Pharmacy and Biomedical Sciences, University of Strathclyde, 161 \\ Cathedral Street, Glasgow G4 0RE, United Kingdom. \\ ${ }^{3}$ Centre for Food Technology and Research, Benue State University Makurdi, Benue State \\ Nigeria. \\ ${ }^{4}$ Department of Chemistry, University of Agriculture Makurdi, PMB 2373, Benue State, \\ Nigeria.
}

Author and co-authors emails:

- Naser Al-Tannak, Email; Dr altannak@hsc.edu.kw

- Ibrahim Khadra, Email: Ibrahim.khadra@strath.ac.uk

- Ngozi Igoli, Email: Ngozi_Igoli@yahoo.com

- John Igoli, Email: igolij@gmail.com

*Corresponding author: Dr. Naser Al-Tannak, Email; Dr_altannak@hsc.edu.kw, Tel: +(965) 2463-6070, 0096599139913 Fax: +(965) 2463-6898 


\begin{abstract}
:
Seeds of Monodora myristica and M. tenuifolia were extracted with hexane and the extracts were subjected to column chromatography, LC-MS and NMR analysis. In addition to masses of previously isolated compounds, other masses corresponding to unidentified compounds from the plants were detected. Using 2D NMR techniques, one of the fractions from $M$. tenuifolia was characterised as a novel 13-(2-butylcyclopropyl)-6,9-dodecadienoic acid. However, none of the compounds detected in LC-MS corresponded to the ones previously identified by GC-MS.
\end{abstract}

Keywords: LC-MS analysis, NMR, Phytochemical analysis, Chromatography, Nigeria 


\section{Introduction}

Monodora myristica and Monodora tenuifolia are the only two Monodora species commonly used as spices in Nigeria. Several phytochemical and GC-MS studies have been carried out on their seed oils (Ukaegbu-Obi et al., 2015, Agiriga A.N, Siwela M, 2018). Previous studies have identified some volatile as well as non-volatile compounds (Ngouana T.K, 2015). The Monodora species are important food spices in most parts of West Africa and Asia. Monodora myristica Geartn. and Monodora tenuifolia Benth (Simo et al., 2018). (family Anonnacea) are widely distributed from Africa to Asia, Central and South America and Australia (Omobuwajo et al., 2003). They are native to West central and East Africa, extending from Sierra Leone to Uganda, Kenya Congo and Angola. Thus Monodora myristica is called African nutmeg. It is one of the most important spice trees of the evergreen forest of West Africa and mostly prevalent in the Southern part of Nigeria (Ravindra and Kallupurackac, 2001). Almost every part of the tree has economic importance. Nutritional value of M. myristica and M. tenuifolia centers on their usefulness as seasonings because of their aromatic flavour. However, the seeds which are embedded in the fruits are of major interest (Uhegbu et al., 2011, Njoku et al, 2012, Ezenwali et al, 2010). The seeds are also used in traditional medicine to relieve tooth ache, dysentery, diarrhea, dermatitis, headache and vermifuge (Ezenwali et al, 2010, Ishola et al., 2016). The plant extracts are reported to have good anti-oxidant activity and could be important in the management and treatment of stress induced diseases (Njoku et al. 2012, Akinwunmi and Oyedapo, 2015, Moukette et al., 2015). Several phytochemical studies to isolate the chemical constituents have been carried out on these plants and other Monodora species (Onyiriuka and Nwaji, 1972; Spiff et al., 1984; Mayunga et al., 2004, Igoli et al., 2011, Ntie-Kang et al., 2014), however, the diversity of the compounds isolated imply there could be much more interesting ones, also taking into account the wide range of bioactivities observed for the plant extracts. Though GC-MS studies of the seed oils (Adewole et al., 2013) have identified myristicin, caffeic acid, safrole, methyl eugenol, catechin, elemicin, quercetin, kaempferol, methyl isoeugenol, eugenol. These studies have not adequately identified all the useful compounds especially the non-volatile ones in the seed oils or non-polar extracts. LC-MS and NMR based metabolomics presents a new way of investigating natural products or plant extracts. It is holistic and unbiased and provides the most functional information about extracts or natural product mixtures. Dereplication studies (Roessner and Dias, 2013) of plant extracts is promising as previously known compounds are easily identified based on the intensities of their NMR or mass spectral peaks. It can also indicate the presence of unknown compounds based on chemical shifts in NMR and unknown elemental composition in MS. This makes for further studies of the extracts or the isolation of such novel compounds. However, no LC-MS studies or column purification which could identify or yield non-volatile and polar compounds have been reported for the seed oils of these plants. The aim therefore is to analyse the hexane extracts and purify by column chromatography the seeds oils of $M$. myristica and M. tenuifolia, and identify the constituents by LC-MS and NMR spectroscopy.

\section{Results and Discussion}

The yield of light brown oils was $42.0 \mathrm{~g}$ from M. myristica and $30.0 \mathrm{~g}$ from M. tenuifolia. Column fractions were oily but the more polar ones yielded white solids. The Characterization of MXHE-29 as 13-(2-butylcyclopropyl)-6,9-Dodecadienoic acid was achieved by NMR analysis and the fraction MXHE-29 on evaporation yielded compound $\mathbf{1}$ as a white solid and it gave a particularly interesting proton nmr spectrum (Figure S1). The proton spectrum indicated it was a single unsaturated fatty acid containing a cyclopropane 
ring (Marcel et al., 1997). It also showed signals for olefinic $\left(\delta_{\mathrm{H}} 5.37\right)$, allylic $\left(\delta_{\mathrm{H}} 2.05\right)$ and bis-allylic $\left(\delta_{\mathrm{H}} 2.79\right)$ protons hence the fatty acid chain must be doubly unsaturated (Knothe and Kenar, 2004, Marcel et al., 1997). A terminal methyl group was observed at $0.90 \mathrm{ppm}$. The cyclopropane methylene protons were the most shielded and were observed at -0.31 and 0.58 while the cyclopropane methine protons were identical and observed at $0.67 \mathrm{ppm}$. The rest of the proton signals was an envelope of $\mathrm{CH}_{2}$ protons from various parts of the fatty acid side chain. The ${ }^{13} \mathrm{C}$-DEPT spectrum (Figure S2) showed one carbonyl carbon at $\delta_{\mathrm{C}} 180.4$, two signals for olefinic doubled bonded carbons at 129.9 and 128.0, cyclopropane carbons at 10.9 (methylene) and 15.8 (methine) and a methyl group carbon at $14.1 \mathrm{ppm}$. Using 2D NMR experiments (Figures S4- S8) such as COSY, TOCSY, HSQC, HMBC and NOESY, the structure was deduced as follows: correlations in the COSY spectrum identified the vicinal protons in the fatty acid chain as well as the germinal ones on the cyclopropane ring. TOCSY was used to identify the spin systems in the various sections of the chain while HMBC was used to connect the sections and the positions of the carboxylic acid group and the olefinic double bonds. The HSQC was used to identify the carbons bearing the protons (Table S2). The $\mathrm{CH}_{3}$ group must be terminal as it showed correlations to only two carbons $(\mathrm{C}-17$ and $\mathrm{C}$ 18 ) in the HMBC and the carboxylic acid group must also be terminal as the two protons $\mathrm{H}-2$ and $\mathrm{H}-3$ that showed correlations to it were vicinal. The olefinic bonds must be on the carboxylic acid part of the chain as H-2 which showed HMBC correlations to it also showed correlations to $\mathrm{C}-4$ and its proton $\mathrm{H}-4$ showed correlations to C-6. Similarly, the cyclopropane ring must be on the terminal $\mathrm{CH}_{3}$ part of the chain as $\mathrm{H}-17$ (attached to $\mathrm{C}-17$ ) and $\mathrm{H}-16$ which are vicinal showed correlations to C-20. Correlations from the bis-allylic protons H-8 confirmed the presence of adjacent double bonds. The linkage of the olefinic section to the cyclopropane ring was confirmed by ${ }^{3} J$ correlations from $\mathrm{H}-12$ to $\mathrm{C}-14$ and $\mathrm{H}-$ 20 to $\mathrm{C}-13$. The compound was thus identified to be 13-(2-butylcyclopropyl)-6,9dodecadienoic acid (1) and confirmed by the mass at 306.2 in its LC-MS (Figure S9). The full chemical shift assignments are given in Table S2 and the structure in Figure S1. The retention times, masses in EI (positive and negative modes) and inferred compounds from the masses are given in Tables S3 and 4. This is based on previously isolated compounds from these plant materials or other Monodora species.

Previous phytochemical reports on the constituents of the seed oils of these plant materials were by GC-MS (Esuoso et al., 2000, Adewole et al., 2013). However, GC-MS reports on the constituents of the seed oils did not identify any of the alkaloids inferred from the LC-MS results (Tables S3 and S4). The major compounds identified under GC-MS were terpenes, fatty acids and triglycerols (Esuoso et al., 2000). The compounds identified under LC-MS in this study were mostly non-volatile and could not have been identified by GC-MS. The novel fatty acid is quite interesting and cyclopropane fatty acids (CFA) have been isolated from several sources such as millipedes (Oudejans, 1971), molluscs (Fenical et al., 1979), molds (Saito and Ochiai, 1998) and a lot of them have been synthesized (Shah et al., 2014, Arai et al., 1983). CFAs are typically found in microorganisms, seed oils of subtropical plants, protozoa and less commonly, within fats and phospholipids produced by animals. They are biosynthesized by methylenation of cis-unsaturated fatty acids via esterification to phospholipids with S-adenosylmethionine and catalyzed by CFA synthases (Shah et al., 2014). 


\section{Experimental}

\subsection{General Experimental Procedures}

The plant seeds were purchased from Markets in Makurdi, Benue State Nigeria and were identified at the Forestry and Wild Life Department, University of Agriculture Makurdi. Voucher specimen were deposited at their herbarium and assigned voucher numbers $\mathrm{UAM} / \mathrm{FH} / 0324$ for $M$. myristica and UAM/FH/0325 for M. tenuifolia. LC-MS was carried out using a Dual source LCMS, an Agilent 6130 with 1200 series LC and UV at $254 \mathrm{~nm}$. NMR spectra were obtained using a Bruker Avance III (400 MHz) spectrophotometer using $\mathrm{CDCl}_{3}$ and TMS as internal standard. Spectra were processed using Mnova software. Column chromatography were carried out using silica gel MN-60 (Macherey-Nagel GmbH \& Co. $\mathrm{KG}$ ) in glass columns eluting gradient wise with hexane, hexane-ethyl acetate, ethyl acetate and ethyl acetate-methanol mixtures. TLC analysis was performed on pre-coated silica gel aluminium plates and spots were visualized using anisaldehyde-sulfuric acid reagent.

\subsection{Extraction of oils}

The ground seeds of M. myristica (200 g) and M. tenuifolia (150 g) were extracted with 500 $\mathrm{mL}$ of hexane each. The solvent was removed using a rotary evaporator to yield the oils.

\subsection{Column chromatography}

About $3.0 \mathrm{~g}$ of the seed oils were separately adsorbed onto silica gel and the resultant slurry dried as much as possible. Glass columns $(45 \mathrm{~cm}$ by $2.0 \mathrm{~cm}$ internal diameter) were packed with $150 \mathrm{~g}$ silica gel in hexane and allowed to settle. Thereafter, the dry slurries of the oils were separately loaded onto the packed columns and eluted gradient-wise using 5\% incremental amounts of ethyl acetate in hexane, ethyl acetate and then methanol in ethyl acetate until 10\% methanol in ethyl acetate. About $25 \mathrm{~mL}$ fractions were collected to obtain 70 fractions altogether. The fractions were allowed to dry, examined by TLC and similar fractions were combined and analysed by NMR. Compound 1 was obtained as fraction MXHE-29 and was not purified further. Its proton NMR showed interesting properties, it was therefore subjected to further 2D NMR analysis to confirm its structure.

\subsection{NMR analysis}

Extracts and fractions were dissolved in $\mathrm{CDCl}_{3}$ and transferred into NMR tubes. Their proton spectra were acquired and examined. Compound 1 (fraction MXHE-29) with interesting proton spectrum was subjected to carbon-13 and 2D (COSY, HMBC, HSQC, DEPT, NOESY and TOCSY) experiments to deduce their structures.

\subsection{LC-MS analysis}

About $1.0 \mathrm{mg}$ of each extract was dissolved in methanol and used for the LC-MS analysis. LC-MS was acquired using the following parameters: Flow rate was $1.00 \mathrm{~mL} / \mathrm{min}$, injection volume was $10.00 \mu \mathrm{L}$. Run time and solvent composition are given in Table S1. For the MS, ionization mode was MM-ES+APCI in both positive and negative polarity and mass range was from 100-1000 mass units.

\section{Conclusion}


Oils from the seeds of Monodora myristica and Monodora tenuifolia were analysed by LCMS and NMR to identify the constituents. The compounds identified by LC-MS were mainly alkaloids of the indole and benzoisoquinoline type while the compounds identified by NMR were long chain unsaturated fatty acids and their triglycerides. A new cyclopropane fatty acid of the type 20:2 (6, 9) was isolated and identified as 13-(2-butylcyclopropyl)-6,9dodecadienoic acid. This study has also confirmed the presence of a CFA in the seed oil of a tropical plant.

\section{Conflict of interest}

The authors declare no conflict of interest.

\section{Acknowledgements}

Authors are grateful to SIPBS, University of Strathclyde Glasgow, Scotland for the NMR and LC-MS analysis. 


\section{References}

Adesomoju A, Ekundayo OO, Eramo TL, Into HR. 1991. Alkaloids of Monodora tenuifolia. Planta Medica. 57(4): 393-4.

Adewole E, Ajiboye BO, Idris OO, Ojo OA, Onikan A, Ogunmodede OT, Adewumi DF. 2013. Phytochemical, Antimicrobial and GC-MS of African Nutmeg (Monodora myristica). International Journal of Pharmaceutical Science Invention. 2(5): 25-32.

Agiriga A.N, Siwela M. 2018. Characterisation of Phytochemicals in Raw and Processed Monodora myristica (Gaertn.) Dunal Seeds by UPLC-MS. Pakistan Journal of Nutrition, 17: 344-354.

Akinwunmi, K, Oyedapo O. 2015. In vitro anti-inflammatory evaluation of african nutmeg (Monodora myristica) seeds. Eur. J. Medi. Plants 8: 167-174

Arai Y, Shimoji K, Konno M, Konishi Y, Okuyama S, Iguchi S, Hayashi M, Miyamoto T, Toda M. 1983. Synthesis and 5-Lipoxygenase Inhibitory Activities of Eicosanoid Compounds. Journal of Medicinal Chemistry. 26: 72-78.

Esuoso KO, Lutz H, Bayer E and Kutubuddin M. 2000. Unsaponifiable Lipid Constituents of Some Underutilized Tropical Seed Oils J. Agric. Food Chem. 48: 231-234

Fenical W, S1eeper, HL, Pau1 VJ, Sta1 1ard MO, Hao H, Sun HH. 1979. Defensive chemistry of navanax and related opisthobranch molluscs. Pure and Applied Chemistry. 51: 1865-1874.

Igoli JO, Gray AI, Clements CJ, Mouad H. 2011. Anti-Trypanosomal activity and cytotoxicity of some compounds and extracts from Nigerian medicinal plants. In Phytochemicals-Bioactivities and impact on Health. Intec Publishers Croatia. Chapter 16: p. 375-388

Ishola, I.O., V.O. Ikumawoyi, G.O. Afolayan and O.J. Olorife. 2016. Antinociceptive and anti-inflammatory properties of hydroethanolic seed extract of Monodora myristica (Annonaceae) in rodents. West Afr. J. Pharm. 27: 22-32.

Knothe, G, Kenar JA. 2004. Determination of the fatty acid profile by ${ }^{1} \mathrm{H}-\mathrm{NMR}$ Spectroscopy. European Journal of Lipid Science and Technology. 106: 88-96

Magoria N, Nyandoro SS, Munissi JJ, Heydenreich EM. 2013. Antimycobacterial and cytotoxicity evaluation of the constituents of Monodora carolinae. Tanz. J. Sci. 39: 12-18

Marcel SF, Jie LK, Mustafa J. (1997) High-Resolution Nuclear Magnetic Resonance Spectroscopy-Applications to Fatty Acids and Triacylglycerols. Lipids 32 (10), 1019-1034.

Moukette B.M, Pieme C.A, Njimou J.R, Nya Biapa C.P, Marco B, Ngogang Y. 2015. In vitro antioxidant properties, free radicals scavenging activities of extracts and polyphenol composition of a non-timber forest product used as spice: Monodora myristica. Biol Res. 48(1): 15 . 
Ngouana, T.K.; Mbouna, C.D.J.; Kuipou, R.M.T.; Tchuenmogne, M.A.T.; Zeuko'o, E.M.; Ngouana, V.; Mallié, M.; Bertout, S.; Boyom, F.F. 2015. Potent and Synergistic Extract Combinations from Terminalia Catappa, Terminalia Mantaly and Monodora tenuifolia Against Pathogenic Yeasts. Medicines. 2, 220-235

Nkunya M H, Makangara JJ, Jonker, SA. 2004. Prenylindoles from Tanzanian Monodora and Isolona Species. Natural Product Research. 18(3): 253-258

Ntie-Kang F, Lifongo LL, Simoben CV, Babiaka SB, Sippl W, Mbaze LM. 2014. The uniqueness and therapeutic value of natural products from West African medicinal plants. Part I: Uniqueness and Chemotaxonomy RSC Advances. 4: 28728-28755

Nwaji MN, Onyiriuka SO, Taylor DAH. 1972. 6-(3-Methylbuta-1 ,3-dienyl)indole from Monodora tenuifolia. Journal Chemical Society Chemical Communications. p 327

Oudejans RCHM, van der Horst DJ, van Dongen JCPM. 1971. Isolation and Identification of Cyclopropane Fatty Acids from the Millipede Graphidostreptus tumuliporus (Karsch) (Myriapoda: Diplopoda) Biochemistry. 10(26): 4938-4941

Simo M.K, Nguepi M.D, Sameza M.L, Toghueo R.K, Fekam F.B, Froldi G. 2018. Cameroonian medicinal plants belonging to Annonaceae family: radical scavenging and antifungal activities, Natural Product Research. 32:17, 2092-2095

Ukaegbu-Obi, K.M., M.O. Meribe and C.E. Odo. 2015. Assessment of antimicrobial activity of aqueous and ethanolic extracts of Monodora myristica (Ehuru) seeds. Mint. J. Pharm. Med. Sci. 4: 1-2.

Roessner U, Dias DA. (Eds) (2013). Metabolomics Tools for Natural Product Discovery: Methods and Protocols. Methods in Molecular Biology 1055. Springer New York. $311 \mathrm{p}$

Saito T, Ochiai H (1998). Fatty Acid Composition of the Cellular Slime Mold Polysphondylium pallidum. Lipids. 33: 327-332

Shah S, White JM, Williams SJ. 2014. Total syntheses of cis-cyclopropane fatty acids: dihydromalvalic acid, dihydrosterculic acid, lactobacillic acid, and 9,10methylenehexadecanoic Acid. Organic and Biomolecular Chemistry. 12: 9427

Spiff AI, Duah FK, Slatkin, DJ, Schiff L Jr. 1984. Alkaloids of Monodora tenuifolia. Planta Medica. 50(5): 455 


\title{
LC-MS analysis of oils of Monodora myristica and Monodora tenuifolia and isolation of a novel cyclopropane fatty acid
}

\author{
Al-Tannak, N.F ${ }^{1,2^{*}}$, Ibrahim Khadra ${ }^{2}$, Igoli N.P and Igoli J.O ${ }^{4}$ \\ ${ }^{1}$ Department of Pharmaceutical Chemistry, Faculty of Pharmacy, Kuwait University, Jamal \\ Abdul Nasser St, Kuwait. \\ ${ }^{2}$ Strathclyde Institute of Pharmacy and Biomedical Sciences, University of Strathclyde, 161 \\ Cathedral Street, Glasgow G4 0RE, United Kingdom \\ ${ }^{3}$ Centre for Food Technology and Research, Benue State University Makurdi, Benue State \\ Nigeria \\ ${ }^{4}$ Department of Chemistry, University of Agriculture Makurdi, PMB 2373, Benue State, \\ Nigeria.
}

\begin{abstract}
Seeds of Monodora myristica and M. tenuifolia were extracted with hexane and the extracts were subjected to column chromatography, LC-MS and NMR analysis. In addition to masses of previously isolated compounds, other masses corresponding to unidentified compounds from the plants were detected. Using 2D NMR techniques, one of the fractions from M. tenuifolia was characterised as a novel 13-(2-butylcyclopropyl)-6,9-dodecadienoic acid. However, none of the compounds detected in LC-MS corresponded to the ones previously identified by GC-MS.
\end{abstract}

Keywords: LC-MS analysis, NMR, Phytochemical analysis, Chromatography, Nigeria

*Corresponding author: Dr. Naser Al-Tannak, Email; Dr_altannak@hsc.edu.kw, Tel: +(965) 2463-6070, 0096599139913 Fax: +(965) 2463-6898 
Table S1. Solvent composition for LC-MS analysis.

\begin{tabular}{|l|l|l|}
\hline Run time (min) & Solvent A (water with 5mM & Solvent B (Acetonitrile with 5mM \\
& ammonium acetate) \% & ammonium acetate) \% \\
\hline 0.00 & 95.0 & 5.0 \\
\hline 1.48 & 95.0 & 5.0 \\
\hline 8.50 & 0.0 & 100.0 \\
\hline 13.50 & 0.0 & 100.0 \\
\hline 16.50 & 95.0 & 5.0 \\
\hline 18.00 & 95.0 & 5.0 \\
\hline
\end{tabular}


Table S2. Chemical shift assignments for compound 1 in $\mathrm{CDCl}_{3}$

\begin{tabular}{|c|c|c|c|c|}
\hline Position & ${ }^{1} \mathrm{H} \delta$ ppm (mult, $J(\mathrm{~Hz})$ & ${ }^{13} \mathrm{C} \delta \mathrm{ppm}$ & $\operatorname{HMBC}\left({ }^{2} J,{ }^{3} J\right)$ & COSY \\
\hline 1 & - & $180.4(\mathrm{C})$ & - & - \\
\hline 2 & $2.36(\mathrm{t}, 7.5)$ & $34.1\left(\mathrm{CH}_{2}\right)$ & $\mathrm{C}-1, \mathrm{C}-3, \mathrm{C}-4$ & $\mathrm{H}-3$ \\
\hline 3 & $1.66(\mathrm{p}, 7.1)$ & $24.6\left(\mathrm{CH}_{2}\right)$ & C-1. C-4 & $\mathrm{H}-2, \mathrm{H}-4$ \\
\hline 4 & $1.36(\mathrm{~m})$ & $29.4\left(\mathrm{CH}_{2}\right)$ & C-6, & H-5, H-6 \\
\hline 5 & $2.05(\mathrm{dq}, 13.1,6.6)$ & $27.2\left(\mathrm{CH}_{2}\right)$ & C-6/7, & H-6, H-7 \\
\hline 6 & $5.37(\mathrm{~m})$ & $129.9(\mathrm{CH})$ & C-5, C-8 & H-5, H-8 \\
\hline 7 & $5.37(\mathrm{~m})$ & $129.9\left(\mathrm{CH}_{2}\right)$ & C-8, C-9 & H-5, H-8 \\
\hline 8 & $2.79(\mathrm{t}, 6.3)$ & $25.7\left(\mathrm{CH}_{2}\right)$ & C-6/7, C-9/10 & H-7, H-9 \\
\hline 9 & $5.37(\mathrm{~m})$ & $128.0\left(\mathrm{CH}_{2}\right)$ & C-11 & $\mathrm{H}-8, \mathrm{H}-11$ \\
\hline 10 & $5.37(\mathrm{~m})$ & $128.0\left(\mathrm{CH}_{2}\right)$ & $\mathrm{C}-11, \mathrm{C}-12$ & $\mathrm{H}-8, \mathrm{H}-11$ \\
\hline 11 & $2.05(\mathrm{dq}, 13.1,6.6)$ & $27.2\left(\mathrm{CH}_{2}\right)$ & C-9/10, C-12 & H-10, H-12 \\
\hline 12 & $1.36(\mathrm{~m})$ & $29.4\left(\mathrm{CH}_{2}\right)$ & C-14, C-13 & H-11, H-13 \\
\hline 13 & $1.17(\mathrm{~m})$ & $28.8\left(\mathrm{CH}_{2}\right)$ & C-15 & H-15, H-17 \\
\hline 14 & $0.67(\mathrm{p}, 5.6,4.7)$ & $15.8(\mathrm{CH})$ & $\mathrm{C}-15$ & H-12, H-20 \\
\hline 15 & $0.67(p, 5.6,4.7)$ & $15.8(\mathrm{CH})$ & C-14, C-20 & H-16, H-20 \\
\hline 16 & $1.17(\mathrm{~m})$ & $28.8\left(\mathrm{CH}_{2}\right)$ & $\begin{array}{l}\mathrm{C}-15, \mathrm{C}-16, \mathrm{C}-17, \\
\mathrm{C}-20\end{array}$ & H-15, H-17 \\
\hline 17 & $1.38(\mathrm{~m})$ & $31.9\left(\mathrm{CH}_{2}\right)$ & C-15, C-19, C-20 & H-16, H-18 \\
\hline 18 & $1.31(\mathrm{~m})$ & $22.8\left(\mathrm{CH}_{2}\right)$ & C-17, C-19 & H-17, H-19 \\
\hline 19 & $0.90(\mathrm{t}, 6.7)$ & $14.1\left(\mathrm{CH}_{3}\right)$ & C-17, C-18 & H-18 \\
\hline 20 & $\begin{array}{l}-0.31(\mathrm{td}, 5.3,3.9) \\
0.58(\mathrm{~m})\end{array}$ & $10.9\left(\mathrm{CH}_{2}\right)$ & C-13, C-14/15 & H-14, H-15 \\
\hline
\end{tabular}


Table S3. LC-MS retention times and masses for Monodora myristica oil

\begin{tabular}{|l|l|l|}
\hline Retention time & $\mathrm{m} / \mathrm{z}$ (mass) & Inference or Compound* \\
\hline $1.376-1.908$ & 145.2 & Indole-5-carbaldehyde \\
\hline 5.020 & 152.2 & Eucalyptol \\
\hline $10.286-10.801$ & 184.2 & 6-(4-oxobut-2-enyl)-indole or 6-(3-methylbuta-1,3-dien- \\
1 -yl)-indole or 3-Dimethylallylindole \\
\hline 5.050 & 236.9 & 9-Hexadecinal \\
\hline 5.384 & 270.2 & Heptadecanoic acid \\
\hline $5.321-5.440$ & 272.2 & Liriodenine \\
\hline 8.408 & 297.0 & Stepharine \\
\hline 10.806 & 340.2 & Laurelliptine \\
\hline $9.323-9.853$ & 367.2 & Annonidine \\
\hline
\end{tabular}

*Based on SciFinder data base hits for compounds previously isolated from Monodora spp 
Table S4. LC-MS retention times and masses for Monodora tenuifolia

\begin{tabular}{|l|l|l|}
\hline Retention time & $m / z$ & Inference or Compound* \\
\hline 0.607 & 145.2 & Indole-5-carbaldehyde \\
\hline 10.275 & 184.2 & $\begin{array}{l}\text { 6-(4-oxobut-2-enyl)-indole or 6-(3-methylbuta-1,3-dien- } \\
\text { 1-yl)-indole }\end{array}$ \\
\hline 5.297 & 273.8 & Liriodenine \\
\hline 6.591 & 288.2 & Kaempferol \\
\hline 8.405 & 297.0 & Stepharine \\
\hline 5.934 & 302.2 & Quercetin \\
\hline 10.804 & 340.2 & Sparsiflorine \\
\hline 10.805 & 341.9 & Magnoflorine or Laurelliptine \\
\hline $9.326-10.787$ & 367.2 & Annonidine \\
\hline 10.804 & 340.2 & Sparsiflorine \\
\hline 10.805 & 341.9 & Magnoflorine or Laurelliptine \\
\hline 12.473 & 560.4 & Cannabisin B \\
\hline $5.325-5.390$ & 306.2 & Compound 1 \\
\hline
\end{tabular}

*Based on SciFinder data base hits for compounds previously isolated from Monodora spp 


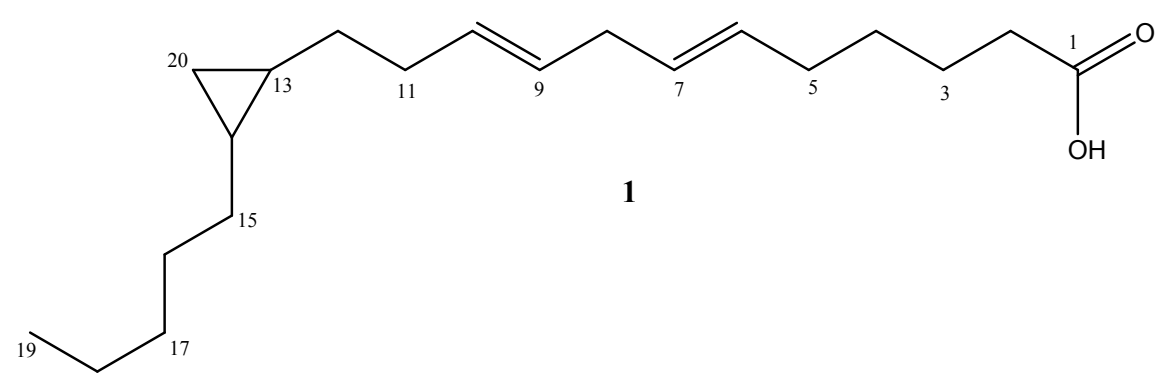

Figure S1. Structure of isolated cyclopropane fatty acid. 


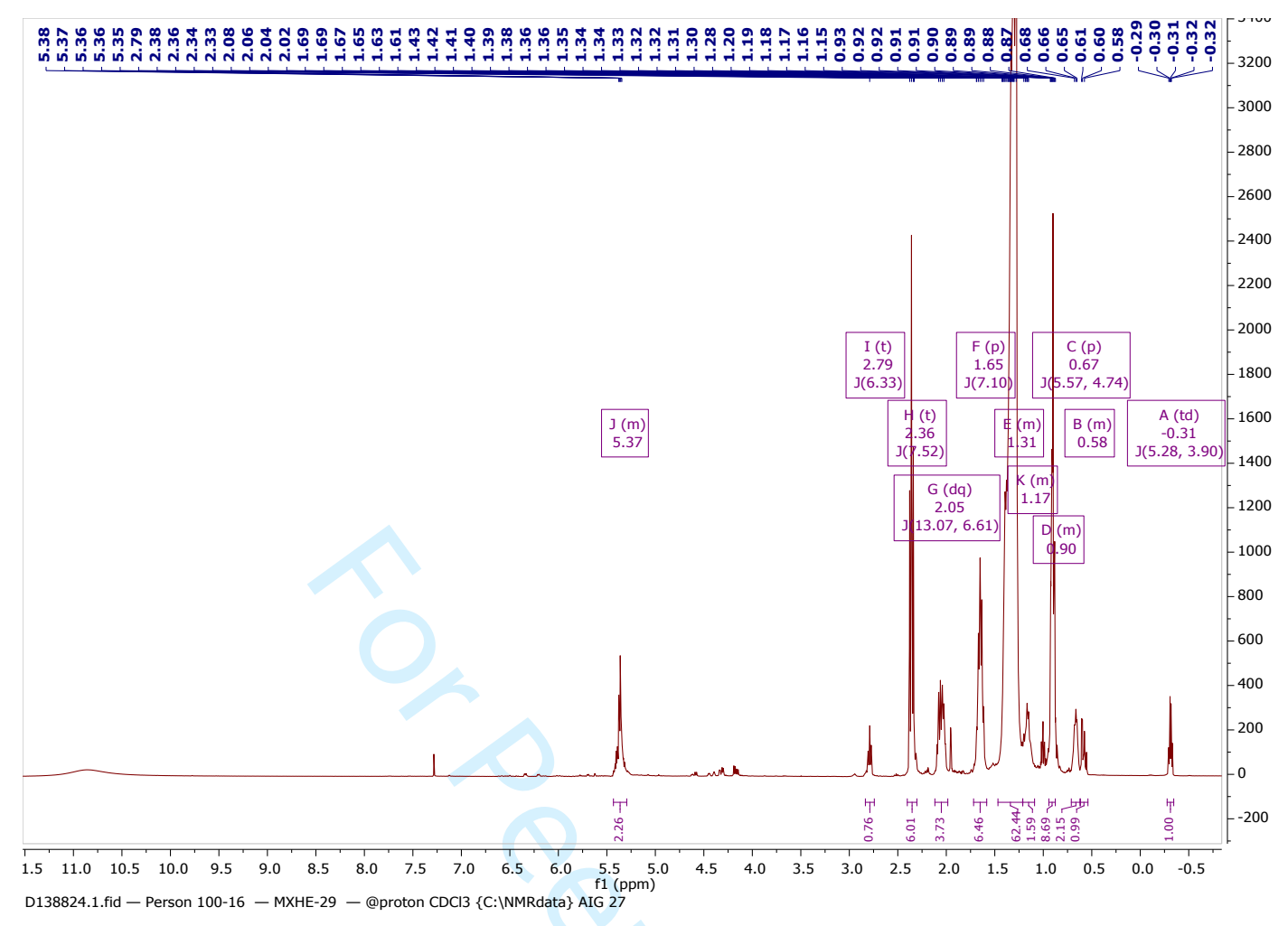

Figure S2. Proton spectrum for compound 1 in $\mathrm{CDCl}_{3}$ 


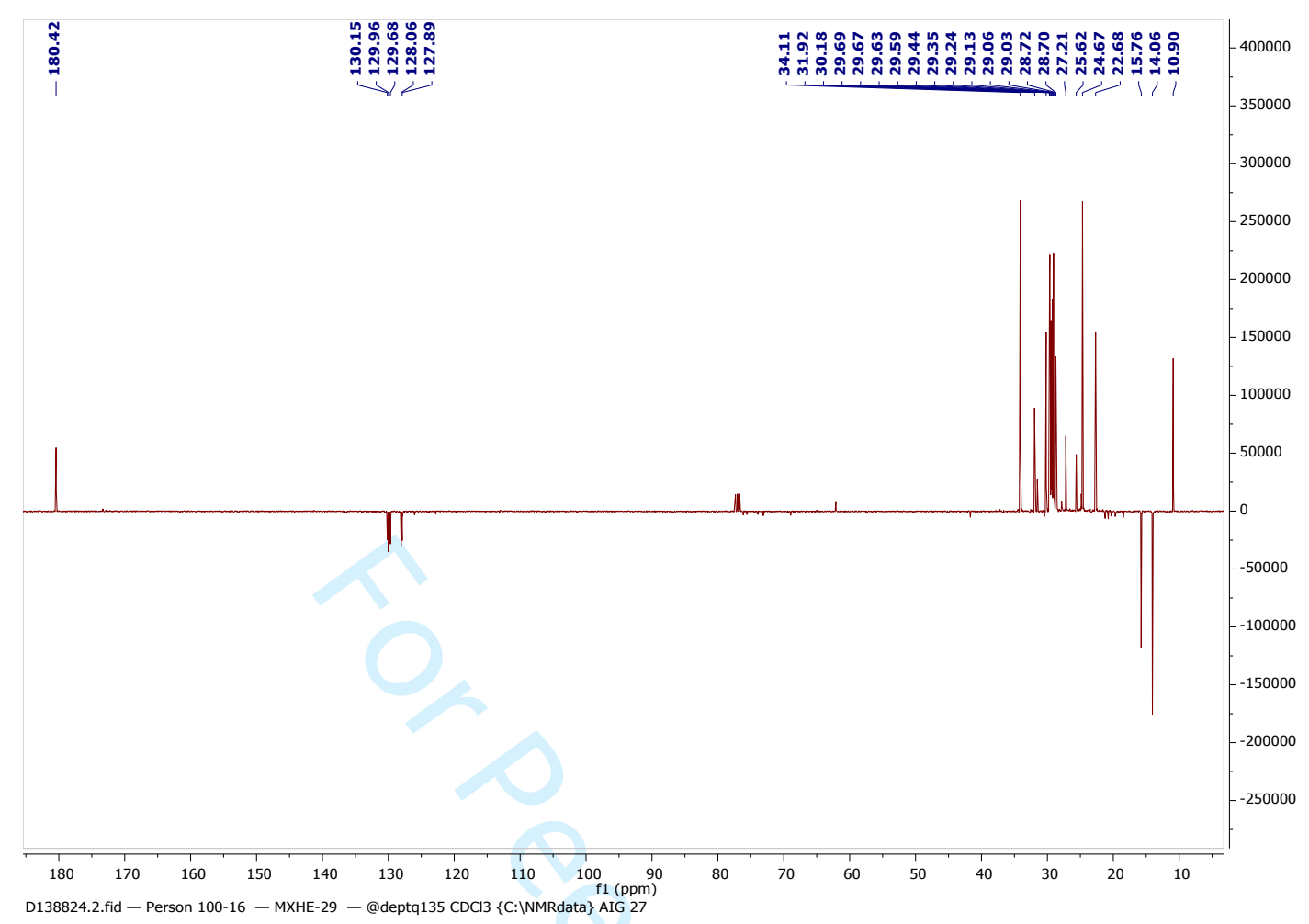

Figure S3. 13-Carbon spectrum for compound 1 in $\mathrm{CDCl}_{3}$ 


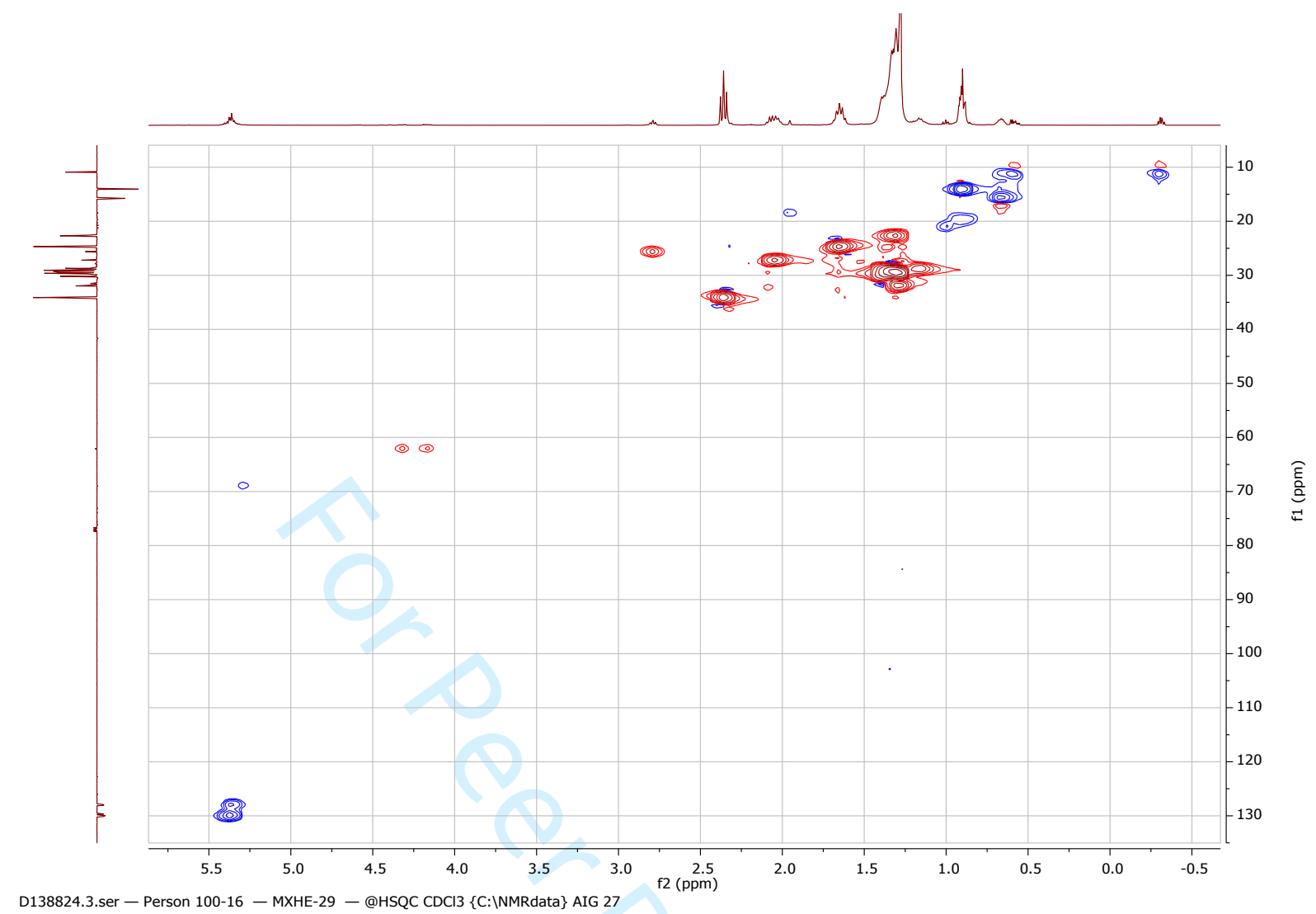

Figure S4. HSQC spectrum for compound 1 in $\mathrm{CDCl}_{3}$ 


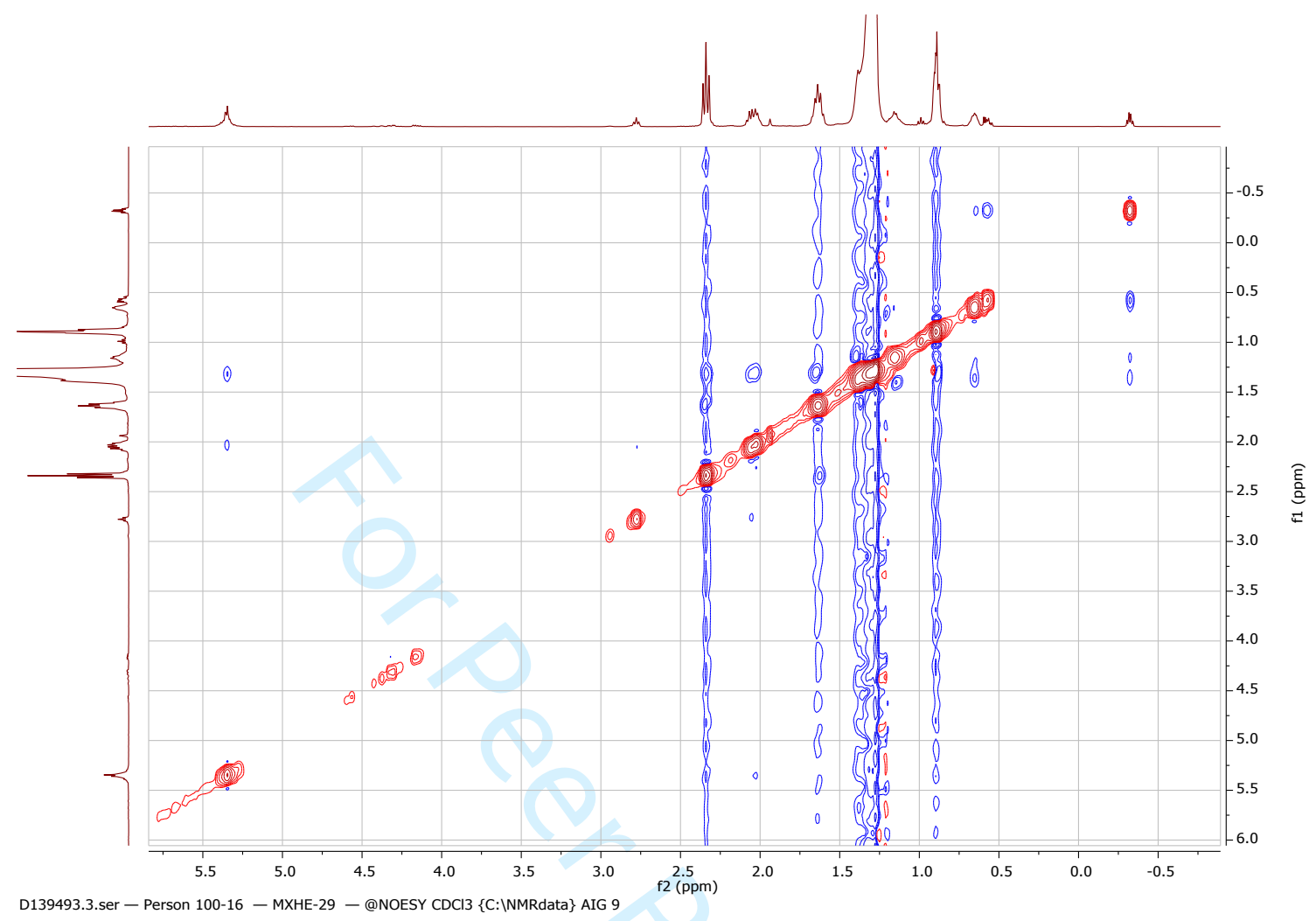

Figure S5. NOESY spectrum for compound 1 in $\mathrm{CDCl}_{3}$ 


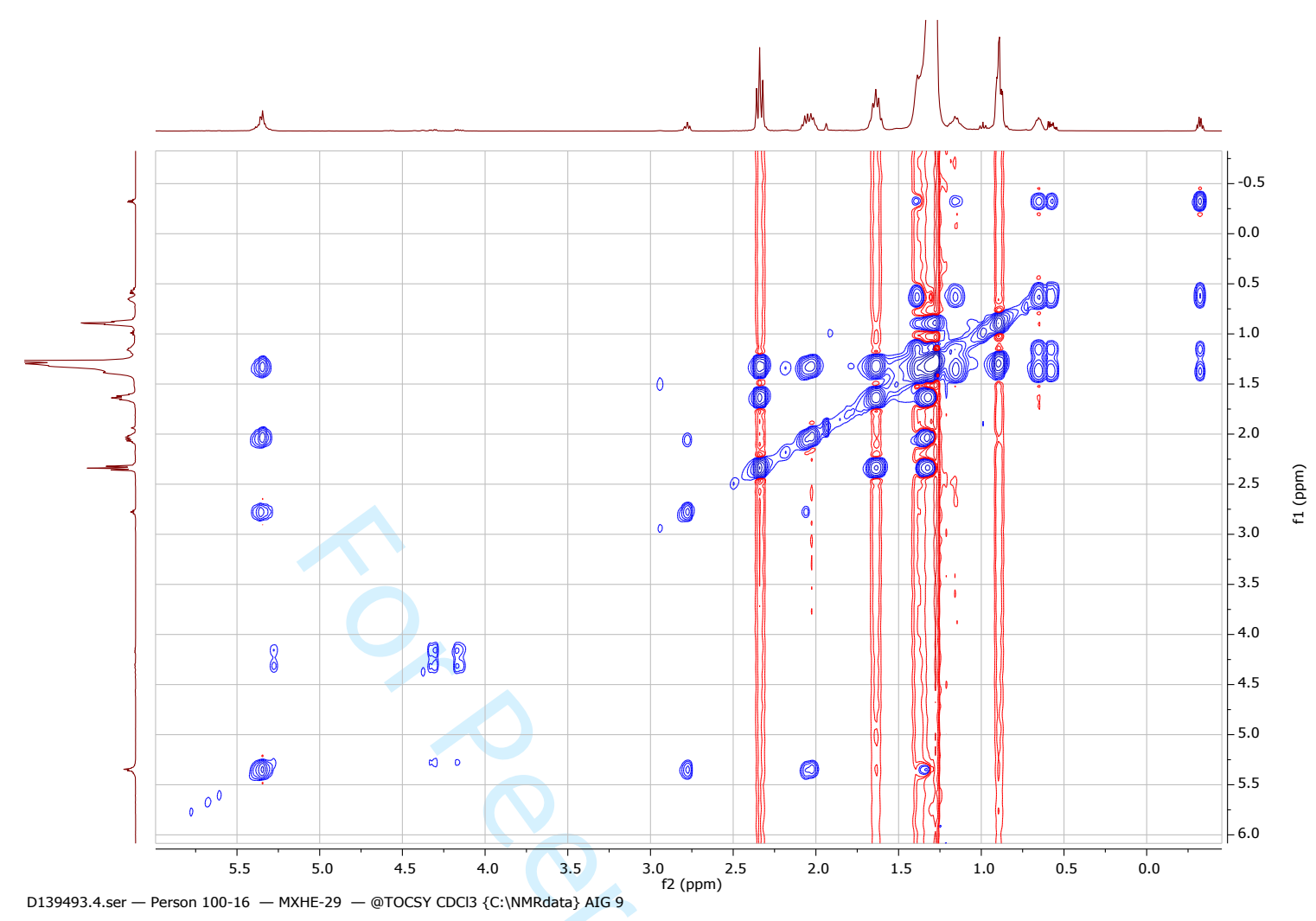

Figure S6. TOCSY spectrum for compound 1 in $\mathrm{CDCl}_{3}$ 


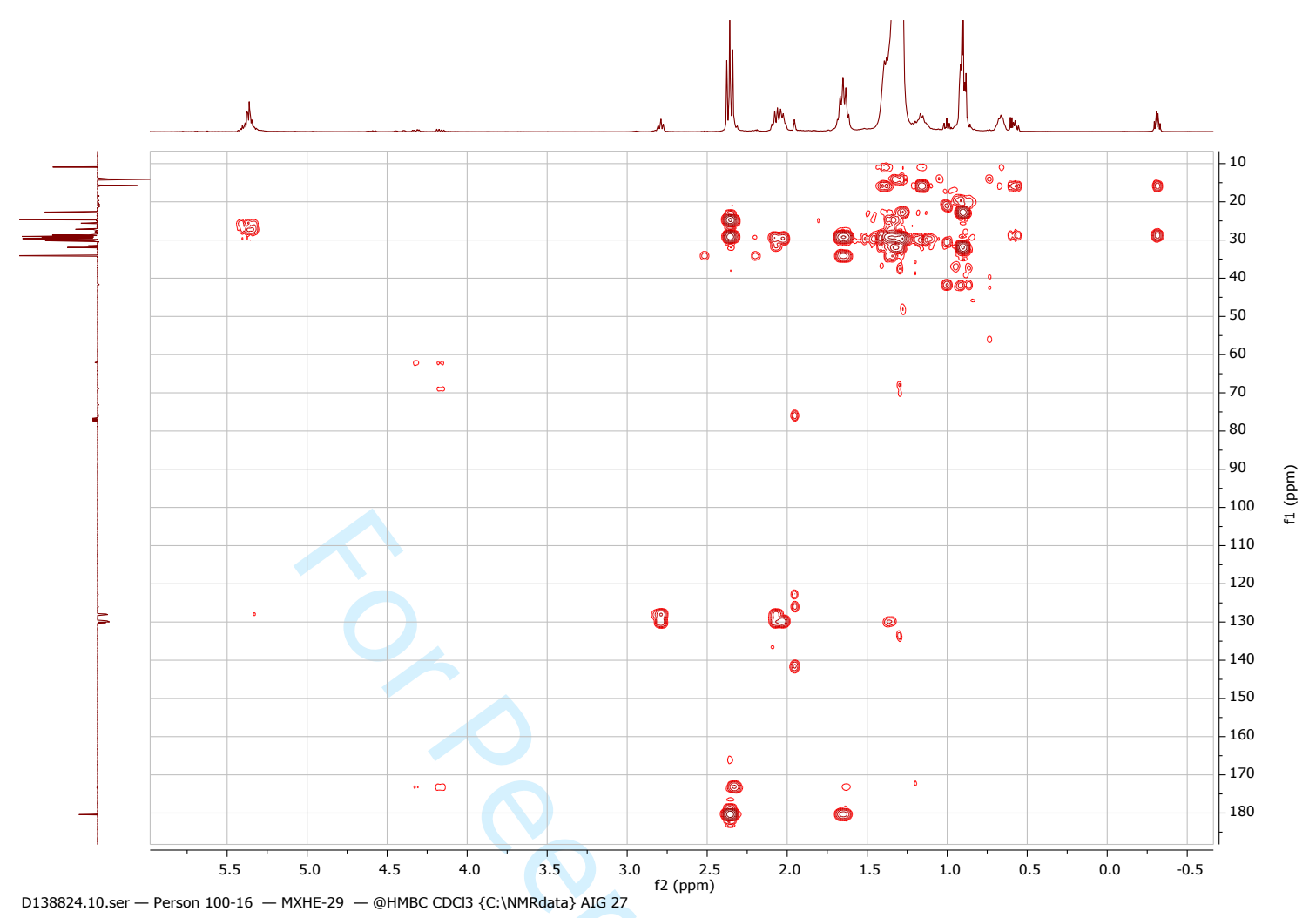

Figure S7. HMBC spectrum for compound 1 in $\mathrm{CDCl}_{3}$ 


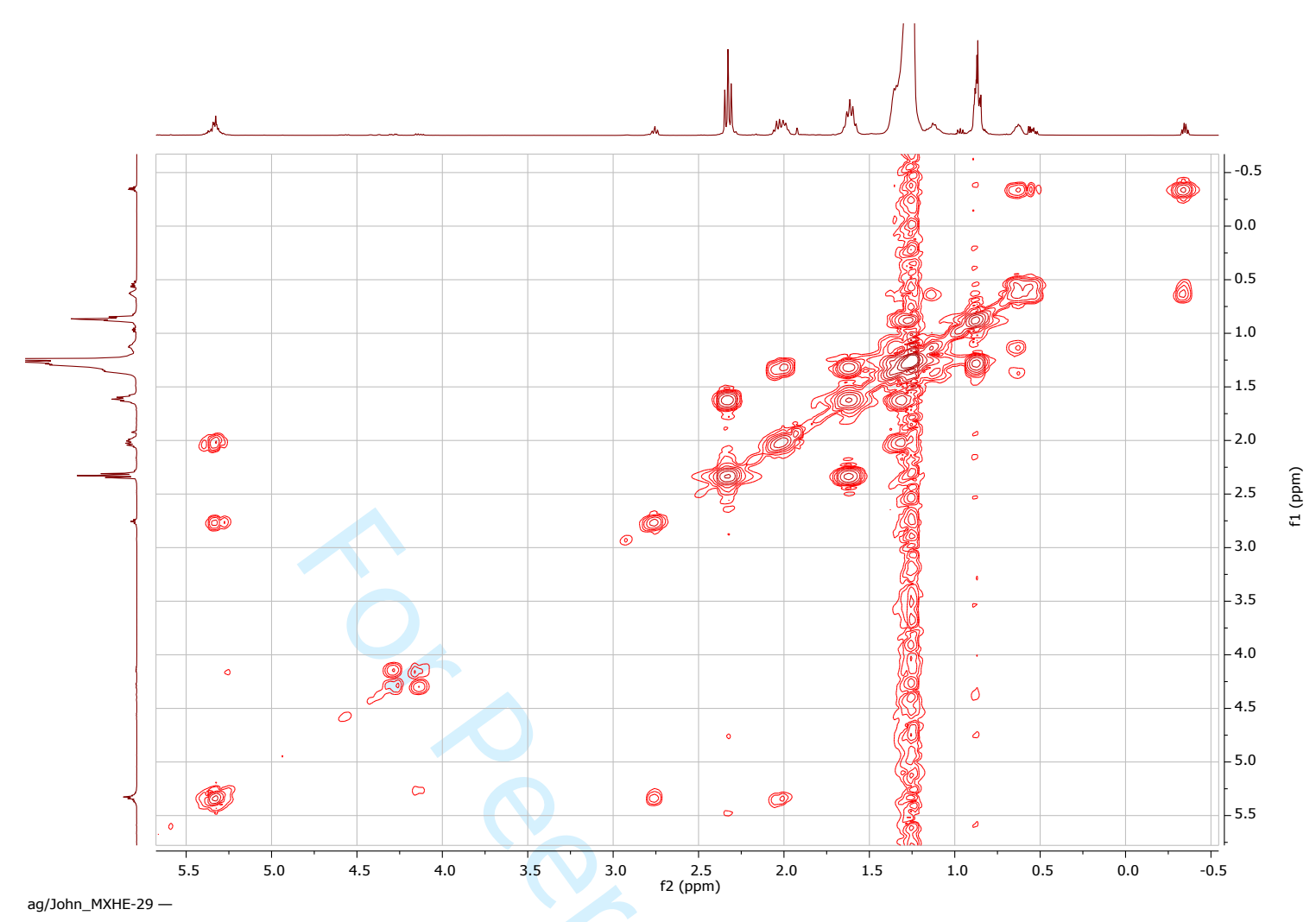

Figure S8. COSY spectrum for compound 1 in $\mathrm{CDCl}_{3}$ 


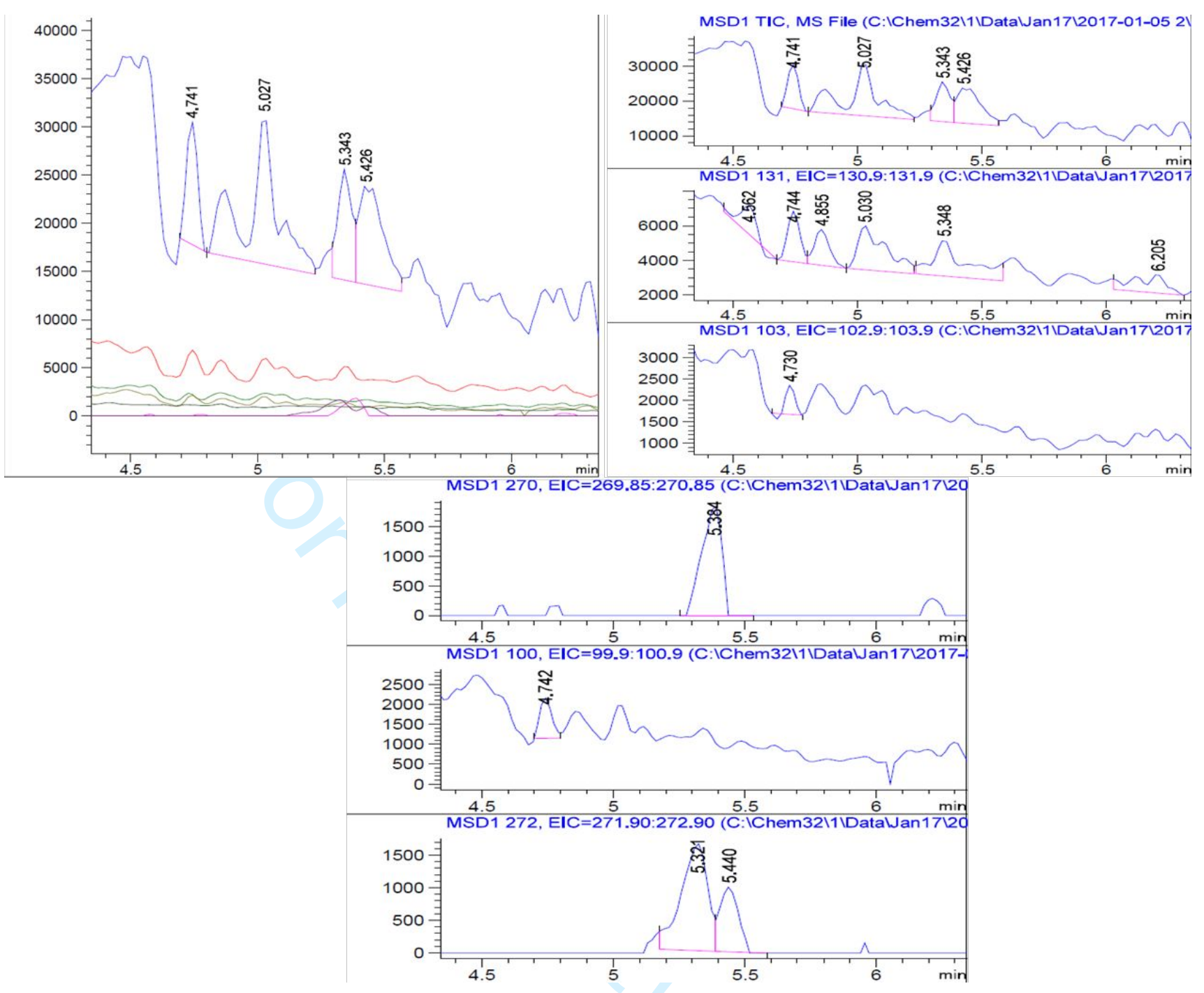

Figure S9. LC-MS total ion chromatogram and spectrum for compound 1 